INPLASY

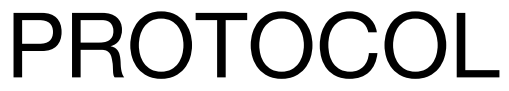

To cite: Qi et al. Efficacy of an novel Oral Janus Kinase 1 Inhibitor Abrocitinib on for patients with Atopic

Dermatitis: a systematic review and meta-analysis. Inplasy

protocol 2020100080. doi: 10.37766/inplasy2020.10.0080

Received: 22 October 2020

Published: 22 October 2020

Corresponding author:

Yanling He

heyanlingdermatology@gmail. com

Author Affiliation:

Beijing Chao-yang Hospital,

Capital Medical University

Support: None.

Review Stage at time of this submission: Preliminary

searches.

Conflicts of interest:

None.

\section{Efficacy of an novel Oral Janus Kinase 1 Inhibitor Abrocitinib on for patients with Atopic Dermatitis: a systematic review and meta-analysis}

Qi, F1; Zhou, S2; Gong, Y3; Zhu, B4; He, Y5.

Review question / Objective: P: adult atopic dermatitis patient; I: abrocitinib; C:placebo; O: the efficacy on EASI, or IGA.

Condition being studied: Atopic dermatitis (AD) is a chronically relapsing inflammatory skin disease that can affect both $2.1 \%$ to $4.9 \%$ of adults and $20 \%$ of children. Nearly one-third of children and half of adults AD patients have moderate or severe disease which has a low response to topical treatment and phototherapy. Biologics are now a new therapeutic method for moderate-to-severe AD patients.

INPLASY registration number: This protocol was registered with the International Platform of Registered Systematic Review and Meta-Analysis Protocols (INPLASY) on 22 October 2020 and was last updated on 22 October 2020 (registration number INPLASY2020100080).

\section{INTRODUCTION}

Review question / Objective: P: adult atopic dermatitis patient; I: abrocitinib; C:placebo; O: the efficacy on EASI, or IGA.

Rationale: Abrocitinib is an oral, once-daily JAK 1 selective inhibitor, which modulates IL-4 and IL-13 and other cytokines that involved in the pathogenesis of AD while sparing JAK 2 inhibition and minimizing the risk for neutropenia and anemia. Oetjen et al. found inhibition of neuronal JAK1 pathways can ameliorate pruritus. Several clinical trials have shown the efficacy of abrocitinib in the treatment of atopic dermatitis, but with few systemic and meta-analysis. 
Condition being studied: Atopic dermatitis (AD) is a chronically relapsing inflammatory skin disease that can affect both $2.1 \%$ to $4.9 \%$ of adults and $20 \%$ of children. Nearly one-third of children and half of adults $A D$ patients have moderate or severe disease which has a low response to topical treatment and phototherapy. Biologics are now a new therapeutic method for moderate-to-severe AD patients.

\section{METHODS}

Search strategy: Two investigators will search the databases like Pubmed, Embase, Cocharne central register of controlled trials (CENTRAL), LILACS, web of science, and gov.trials.

Participant or population: Moderate-tosevere adult atopic dermatitis patients.

Intervention: Abrocitinib.

Comparator: Placebo.

Study designs to be included: Phase II and phase III randomized clinical trials.

Eligibility criteria: Random controlled trials, with population of moderate-to-severe atopic dermatitis patients, above 18 years old, in English and with full-text accessible.

Information sources: Electronic databases, trial registers, and grey literature.

Main outcome(s): The efficacy of abrocitinib in the treatment of dermatology, in EASI, IGA comparing to placebo.

Additional outcome(s): Efficacy of abrocitinib in a different dose and the safety of this biologics.

Data management: Two investigators will extract the data.

Quality assessment / Risk of bias analysis: Cochrane Risk of Bias Tool for Randomized Controlled Trials will be used to evaluated the literature quality, three groups of study quality will be defined as high, middle, and low. A funnel plot and Egger test will be used to access the publication bias, and Eggers test overruled when results diverged between methods.

Strategy of data synthesis: We will perform a random effect analysis due to considerable heterogeneity and $95 \%$ confidence intervals will be used. statistical heterogeneity will be assessed through 12 statistic.

Subgroup analysis: Subgroup analyses were only performed if there were sufficient data. Subgroup analysis will be performed according to different dosage.

Sensibility analysis: Given considerable heterogeneity, random effect analysis will be used.

\section{Language: English.}

\section{Country(ies) involved: China.}

Keywords: Atopic dermatitis, abrocitinib, JAK inhibitors.

Contributions of each author:

Author 1 - Fei Qi - search the literature, data extraction, data analysis and write the draft.

Author 2 - Shuying Zhou - search the literature, data extraction, data analysis.

Author 3 - Yue Gong - review the literature, assess the literature based on inclusion criteria.

Author 4 - Binghua Zhu - review the literature, assess the literature based on inclusion criteria.

Author 5 - Yanling He - visualization, software, funding, supervising, conception. 